\section{Morbidity and Mortality Rates for Peripheral Vascular Surgery}

\section{To the Editor:}

I have been asked by the Surgery Committee and the Infection Control Committee of my hospital to obtain some statistics and standards for comparison. We are looking for morbidity and mortality rates for peripheral vascular surgery. We particularly want to know the expected "Returns to Surgery," the "Infection Rates," the number of amputations to expect, and other information that may be pertinent.

This kind of information has not been available from the Public Health Department, The Peripheral Vascular Surgery Library at either UCLA or USC Medical Centers, CDC, or local hospital Medical Records Departments.

I will certainly appreciate any information you can provide.

Pat McCabe, RN Infection Control and Quality Assurance Coordinator Bellwood General Hospital Bellflower, California

Dr. Harold Laufman and Drs. Daniel S. Rush and Ronald L. Nichols were asked to respond to Ms. McCabe's query.

One reason for the lack of success in getting "average" or "standard" statistics on morbidity and mortality rates for peripheral vascular surgery is that vascular surgery embraces so many widely divergent facets.

For example, if you want figures on incidence of infection following implantation of a peripheral vascular prosthesis, published statistics show a range from 1.2 to 6.7 . But this is deceiving, because if those figures are broken down into anatomical loca- tions, you will find that when implanted prostheses are placed in the inguinal region, the incidence of infection is closer to $15 \%$ to $20 \%$.

As long as foreign substance is used for vascular grafts it will be impossible to reduce the infection-rate figure to zero. It is well-known that the implantation of foreign material enhances the chances for infection almost regardless of how few bacteria are seeded in the wound at the time of surgery. There are invariably some bacteria seeded at any operation. Infection following vascular surgery without the implantation of foreign material is of course, much less likely to occur than in the presence of implanted foreign material.

In any case, the incidence of infection is also directly proportional to the age, nutritional status, and medical status of the population undergoing the surgery. Moreover, one inevitably finds a higher incidence of infection in teaching hospitals with a large service than in smaller private hospitals in which the surgeon is solely responsible for the entire management of the case.

The use of prophylactic and perisurgical antibiotics is also known to affect infection rates. Similarly, the diligence with which the skin is prepared, and how meticulously the operative technique is carried out must not be overlooked.

As to the incidence of amputation in a vascular surgical service, much depends upon whether the amputations are the end result of failure of efforts at revascularization or salvage, or whether the amputations are performed for frank gangrene or unsalvageable limbs of patients admitted with the indications for amputation. These points, in turn, are dependent on the kind of patient population as well as all other factors mentioned above. Mortality rates, likewise, must be based upon such detailed information, or they are useless. For these reasons most of the published data on amputations are not worth quoting here.

I would refer you to an excellent book edited by Bernhard and Towne entitled Complications in Vascular Surgery published by Grune and Stratton, 1980.

\author{
Harold Laufman, MD, PhD, FACS \\ Surgical Consultant \\ New York, New York
}

Unfortunately, as stated, this information is often not readily found in a single source and differences in reporting make interpretation or generalization difficult. There are, however, fairly well-accepted standards used by vascular surgeons in evaluating their work which are presented here. Peripheral vascular operations vary in morbidity and mortality, depending upon differences in: 1) patient population, 2) age and risk factors, 3) indications for surgery, 4) type of procedure, 5) surgical technique, and 6) graft material used.

Elective aortic reconstruction (aneurysmectomy or aorto-femoral bypass), should carry a $1 \%$ to $3 \%$ operative mortality; however, operations for ruptured abdominal aortic aneurysms often report a $40 \%$ to $50 \%$ mortality. It is generally accepted that other peripheral vascular procedures carry a $1 \%$ to $2 \%$ mortality, or less. Overall morbidity, including complications discussed below and others, should be less than $5 \%$ to $10 \%$.

Early graft thrombosis (within 30 days) should be less than $5 \%$. Of these early graft failures, $80 \%$ to $90 \%$ will be due to technical or judgmental errors. Graft thrombectomy and revision are usually required to avoid limb loss in this situation. Use of intraoperative arteriography or Doppler ultrasound examination has been shown to reduce the incidence of technical errors. Late graft failures are often due to progression of atherosclerosis or intimal hyperplasia at the distal anastomosis.

Peripheral vascular operations are 